PROCEEDINGS OF THE AMERICAN MATHEMATICAL SOCIETY

Volume 124, Number 1, January 1996

\title{
FACTORIAL DOMAINS
}

\author{
CLIFFORD S. QUEEN
}

(Communicated by Wolmer V. Vasconcelos)

\begin{abstract}
We give a simple characterization of factorial domains. We also characterize almost factorial domains and Krull domains with finite cyclic class group.
\end{abstract}

\section{INTRODUCTION}

Let $A$ be an integral domain and $K$ its field of fractions. We say that $A$ is a factorial domain if there exists a subset $P$ of $A$ not containing 0 such that any nonzero $a$ in $A$ can be written uniquely up to order as a product

$$
a=u \prod_{p \in P} p^{n(p)}
$$

where $u$ is a unit of $A$ and the $n(p)$ are nonnegative integers with $n(p)=0$ for all but finitely many $p$.

The two most well-known examples of factorial domains are $Z$, the ring of integers, and $F[X]$, the ring of polynomials in one variable $X$ over a field $F$. Both of these rings are principal ideal domains (PIDs) and all such rings are known to be factorial (see [6]). Let $Q$ denote the field of rational numbers. In 1928 Hasse proved (see [4]) the following result:

Theorem 1. A is a PID if and only if there is a map $N: K \longrightarrow Q$ satisfying the following properties:

(1) $N(x) \geq 0$ for all $x$ in $K$ and $N(x)=0$ if and only if $x=0$.

(2) $N(x y)=N(x) N(y)$ for all $x$ and $y$ in $K$.

(3) $N(a) \in Z$ for all $a \in A$.

(4) For a in $A, N(a)=1$ if and only if $a$ is a unit of $A$.

(5) Given an $x$ in $K$ such that $x$ is not in $A$ there exist $a$ and $b$ in $A$ with $0<N(a x-b)<1$.

We prove a more general result (Theorem 2) in section 2. However a sketch of the proof of Theorem 1 is as follows: Suppose $A$ is an integral domain and there is a map $N: K \longrightarrow Q$ satisfying the above four properties. To show that $A$ is a PID let $\mathcal{I}$ be a nonzero ideal in $A$ and $N(d)$ minimal over all nonzero elements $d$ of $\mathcal{I}$. We claim that $\mathcal{I}=A d=(d)$, since if $e$ is in $\mathcal{I}$ and $d$ does not divide $e$, then $e / d$ is not in $A$ and so there exist $a, b \in A$ so that $0<N((e / d) a-b)<1$. Thus $e a-b d$ is a nonzero element of $\mathcal{I}$ and $N(e a-b d)<N(d)$, which is impossible.

Received by the editors February 18, 1994 and, in revised form, June 4, 1994.

1991 Mathematics Subject Classification. Primary 13M15.

(C)1996 American Mathematical Society 
Next suppose that $A$ is a PID. To construct a map $N: K \longrightarrow Q$ satisfying the four properties of our theorem, let $P$ be a set consisting of one irreducible element from each associate class of irreducible elements. Then set $N(0)=0$ and if $a$ is a nonzero element of $A$ we set $N(a)=2^{w(a)}$, where $w(a)$ is the number of factors of elements of $P$ in a factorization of $a$, counting multiplicity. Finally, if $x$ is a nonzero element of $K$, then there exist $a, b \in A$ such that $a b \neq 0$ and $x=a / b$ and so we set $N(x)=N(a) / N(b)$. One then completes the proof by showing that $N$ satisfies properties (1)-(5) above.

Of course a factorial domain need not be a PID. Examples are the ring of polynomials in one variable over the integers and the ring of polynomials in more than one variable over a field. We achieve one objective of this paper in section 2 by proving Theorem 2, which is a characterization of factorial domains that generalizes Theorem 1 . In section 3 we generalize Theorem 1 in yet another direction by characterizing Krull domains with finite cyclic class groups.

\section{FACTORIAL DOMAINS}

Let $A$ be an integral domain and $K$ its field of fractions. Recall that an ideal of $A$, usually referred to as a fractional ideal, is an $A$-module in $K$ of the form $x \mathcal{I}$, where $\mathcal{I}$ is an $A$-module contained in $A$ and $x$ is in the multiplicative group of $K$, denoted by $K^{*}$. A divisorial ideal $\mathcal{A}$ is an intersection of principal ideals, i.e. there is a subset $S$ of $K^{*}$, such that

$$
\mathcal{I}=\bigcap_{x \in S} A x
$$

If $\mathcal{I}$ and $\mathcal{J}$ are nonzero ideals, then the following are a list of known results whose proofs can be found in Fossum (see [2]):

(1) All divisorial ideals are of the form $(A: \mathcal{I})=\{x \in K \mid x \mathcal{I} \subseteq A\}$.

(2) If $\mathcal{I} \subseteq \mathcal{J}$, then $(A: \mathcal{J}) \subseteq(A: \mathcal{I})$.

(3) $(A:(A: \mathcal{I}))$ is the smallest divisorial ideal containing the ideal $\mathcal{I}$.

If $a, b \in A$ and $b \neq 0$, then $b$ divides $a$ (in notation $b \mid a$ ) means there exists $c \in A$ so that $a=b c$. Now the units of $A$ are simply those $u$ in $A$ so that $u \mid 1$. Further we say that a nonunit $b$ properly divides $a$ if $a=b c$ and $c$ is not a unit of $A$. A nonzero element $c$ of $A$ is determined up to a unit multiple as the greatest common divisor of two elements $a$ and $b$ of $A$, if $c|a, c| b$ and for any $d \in A$ such that $d \mid a$ and $d \mid b$, then $d \mid c$. Our notation is $c=\operatorname{gcd}(a, b)$. It is shown in Jacobson (see [6]) that $A$ is a factorial domain if and only if any two nonzero elements of $A$ have a gcd and the ascending chain condition holds for integral principal ideals of $A$. This last condition is referred to as ACCP and simply means that there are no infinite ascending chains of integral principal ideals.

Definition 1. A mapping $N: K \longrightarrow Q$ is said to be a norm on the pair $(A, K)$ if the following properties hold:

(1) $N(x) \geq 0$ for all $x$ in $K$ and $N(x)=0$ if and only if $x=0$.

(2) $N(x y)=N(x) N(y)$ for all $x$ and $y$ in $K$.

(3) $N(a) \in Z$ for all $a \in A$.

(4) For $a$ in $A, N(a)=1$ if and only if $a$ is a unit of $A$.

Theorem 2. An integral domain $A$ is a factorial domain if and only if there is a norm $N$ on the pair $(A, K)$ satisfying the following condition: Given $a, b \in A$ 
such that $a$ does not divide $b$ and $b$ does not divide $a$, there exists a nonzero $c \in$ $(A:(A:(a, b)))$ with $N(c)<\min (N(a), N(b))$.

Proof. Suppose that there is a norm $N$ on the pair $(A, K)$ satisfying the above condition. As we saw above it suffices to prove that any two nonzero elements of $A$ have a gcd and that ACCP holds for $A$.

(gcd) Let $a, b \in A$ with $a b \neq 0$. Let $c$ be a nonzero element of $(A:(A:(a, b)))$ of smallest norm, $N(c)$. We claim that $(A:(A:(a, b)))=c A$. Suppose $e \in(A:$ $(A:(a, b)))$ and $c$ does not divide $e$; then $e$ cannot divide $c$ because $N(c) \leq N(d)$ and if $e \mid c$, then $N(c / e)=1$, i.e. $e$ would be a unit times $c$, which is not possible by our assumption. Now there exist a nonzero $f \in(A:(A:(e, c)))$ such that $N(f)<\min (N(e), N(c))=N(c)$. However because $(e, c) \subseteq(A:(A:(a, b)))$, we have that $(A:(A:(A:(a, b)))) \subseteq(A:(e, c))$ and thus

$$
(A:(A:(e, c))) \subseteq(A:(A:(A:(A:(a, b))))=(A:(A:(a, b))),
$$

contradicting the minimality of $N(c)$. Now to show that $c=\operatorname{gcd}(a, b)$, we note that $c \mid a$ and $c \mid b$. Next if $d \in A$ such that $d \mid a$ and $d \mid b$, i.e. $(a, b) \subseteq A d$, then since $A d$ is divisorial and $(A:(A:(a, b)))$ is the smallest divisorial ideal containing $(a, b)$, we have that $c A=(A:(A:(a, b))) \subseteq A d$, i.e. $d \mid c$.

(ACCP) If we had an infinite sequence of $a_{1}, a_{2}, \ldots$ so that $A a_{i} \subset A a_{i+1}$, for $i \geq 1$, then we would have an infinite strictly decending chain of positive integers $N\left(a_{1}\right)>N\left(a_{2}\right)>\ldots$, which is clearly impossible.

Now suppose that $A$ is a factorial domain. Let $P$ consist of one irreducible element from each associate class of irreducible elements. Then if $a$ is a nonzero element of $A$, we write

$$
a=u \prod_{p \in P} p^{n(p)}
$$

where $u$ is a unit of $A$ and the $n(p)$ are nonnegative integers with $n(p)=0$ for all but finitely many $p$. Here we set $N(a)=2^{\sum_{p \in P} n(p)}$. We set $N(0)=0$ and extend $N$ by multiplicativity to all of $K$. Now it is easy to see that $N$ is a norm on the pair $(A, K)$.

Next suppose $a, b \in A$ such that $a$ does not divide $b$ and $b$ does not divide $a$. If $c=\operatorname{gcd}(a, b)$, then $c A=(A:(A:(a, b)))$ and since $c$ properly divides $a$ and $b$, we must have that $N(c)<\min (N(a), N(b))$.

\section{KRull domains}

Let $D(A)$ denote the collection of nonzero divisorial ideals of $A$ in $K$. We partially order $D(A)$ by set-theoretic containment and introduce a binary operation as follows: If $\mathcal{I}, \mathcal{J} \in D(A)$, then $\mathcal{I} \circ \mathcal{J}=(A:(A: \mathcal{I} \mathcal{J}))$. Now according to Fossum in ([2]) this order and binary operation makes $D(A)$ a lattice-ordered commutative monoid with $A$ as identity. Further, if $x \in K^{*}$ and $\mathcal{I} \in D(A)$, then $\mathcal{I} \circ A x=\mathcal{I} x$ and in fact the principal ideals $P(A)$ form a subgroup of $D(A)$. Let $D(A)^{+}$denote the integral divisorial ideals and $C L(A)=D(A) / P(A)$ the monoid of divisorial ideal classes. We call $A$ a Krull domain if, under the above defined binary operation, $D(A)$ is a group and the elements of $D(A)^{+}$satisfy the ascending chain condition, i.e. there is no infinite sequence of elements of $D(A)^{+},\left\{\mathcal{I}_{i}\right\}_{i=1}^{\infty}$, where $\mathcal{I}_{i+1}$ properly contains $\mathcal{I}_{i}$.

Suppose $A$ is a Krull domain. Let $\Im$ denote the set of prime divisorial ideals in $D(A)^{+}$; then it is known (see [9]) that $D(A)$ is the free abelian group on the 
elements of $\Im$. So in particular if $\mathcal{I}$ is in $D(A)$ we can write

$$
\mathcal{I}=\prod_{\mathcal{P} \in \Im}^{\circ} \mathcal{P}^{\left(v_{\mathcal{P}}(\mathcal{I})\right)}
$$

where the $v_{\mathcal{P}}(\mathcal{I})$ 's are integers uniquely determined by $\mathcal{I}$ and $\mathcal{P}$ and are zero for all but finitely many $\mathcal{P}$. Our notation needs a little explanation: We write $\prod^{\circ}$ and $\mathcal{P}^{(n)}$, for $n$ an integer, to indicate that our binary operation is o. We can now define a function on $D(A)$ as follows: Set $N(A)=1$ and if $\mathcal{I} \neq A$ is in $D(A)$ we set $N(\mathcal{I})=2^{l(\mathcal{I})}$, where $l(\mathcal{I})=\sum_{\mathcal{P} \in \Im} v_{\mathcal{P}}(\mathcal{I})$. Now let $Q^{+}$and $Z^{+}$denote the positive rationals and the positive integers respectively. We have defined a map $N: D(A) \longrightarrow Q^{+}$. It follows then that $N$ satifies the properties:

(1) For $\mathcal{I}, \mathcal{J}$ in $D(A), N(\mathcal{I} \circ \mathcal{J})=N(\mathcal{I}) N(\mathcal{J})$.

(2) If $\mathcal{I}$ is $D(A)^{+}$, then $N(\mathcal{I})$ is in $Z^{+}$and $N(\mathcal{I})=1$ if and only if $\mathcal{I}=A$.

Definition 2. Now let $A$ be any integral domain. A mapping $N: D(A) \longrightarrow Q^{+}$ is called a norm map on $D(A)$ if it satisfies the following properties:

(1) For $\mathcal{I}, \mathcal{J}$ in $D(A), N(\mathcal{I} \circ \mathcal{J})=N(\mathcal{I}) N(\mathcal{J})$.

(2) If $\mathcal{I}$ is $D(A)^{+}$, then $N(\mathcal{I})$ is in $Z^{+}$and $N(\mathcal{I})=1$ if and only if $\mathcal{I}=A$.

Definition 3. A Krull domain is said to be almost factorial if $C L(A)$ is a torsion group, i.e. every element of $C L(A)$ is of finite order.

A generalization of Theorem 2 is the following.

Theorem 3. An integral domain $A$ is an almost factorial domain if and only if there is a norm $N$ on $D(A)$ with the property: Given $\mathcal{I} \neq A$ in $D(A)^{+}$, there exist a positive integer $k$ and an element $x$ in $K^{*}$ so that $\mathcal{I}^{(k)} x$ is in $D(A)^{+}$and $N\left(\mathcal{I}^{(k)} x\right)<N(\mathcal{I})$.

Proof. Suppose $A$ is an almost factorial domain. We saw above that there is a norm on $D(A)$. Let $N$ be any norm on $D(A)$. If $\mathcal{I} \neq A$ is in $D(A)$, then there exists a positive integer $k$ so that $\mathcal{I}^{(k)}=(y)=A y$ is a principal ideal, $y$ a nonzero element of $A$. Hence $\mathcal{I}^{(k)} y^{-1}=A$ is in $D(A)^{+}$and $N(A)=1<N(\mathcal{I})$.

Now suppose that $A$ is an integral domain and there is a norm $N$ on $D(A)$. This alone guarantees the ascending chain condition on $D(A)^{+}$, since if we have an infinite chain of elements of $D(A)^{+},\left\{\mathcal{I}_{i}\right\}_{i=1}^{\infty}$, where $\mathcal{I}_{i+1}$ properly contains $\mathcal{I}_{i}$, then $\left\{N\left(\mathcal{I}_{i}\right)\right\}_{i=1}^{\infty}$ would be an infinite descending chain of positive integers, which is impossible. Next we assume the property: Given $\mathcal{I} \neq A$ in $D(A)^{+}$, there exist a positive integer $k$ and an element $x$ in $K^{*}$ so that $\mathcal{I}^{(k)} x$ is in $D(A)^{+}$and $N\left(\mathcal{I}^{(k)} x\right)<$ $N(\mathcal{I})$. We will show that $C L(A)$ is a torsion group from which it follows that $D(A)$ is a group and thus $A$ is a Krull domain and every element of $C L(A)$ has finite order. Proceeding by induction we note first that $A$ is principal and we suppose that $\mathcal{I}$ is in $D(A)^{+}, \mathcal{I} \neq A$ and for any $\mathcal{J}$ in $D(A)^{+}$such that $N(\mathcal{J})<N(\mathcal{I})$, we have that some power of $\mathcal{J}$ is principal. Then there exist a positive integer $k$ and an element $x$ in $K^{*}$ so that $\mathcal{I}^{(k)} x$ is in $D(A)^{+}$and $N\left(\mathcal{I}^{(k)} x\right)<N(\mathcal{I})$. Hence there is a positive integer $m$ so that $\left(\mathcal{I}^{(k)} x\right)^{(m)}=(z)$ is a principal ideal, i.e. $\mathcal{I}^{(k m)}=\left(z x^{-m}\right)$.

Theorem 4. Suppose $A$ is an integral domain, $N$ is a norm map on $D(A)$ and there is a class $C$ in $C L(A)$ with the following property: given $\mathcal{I}$ in $D(A)^{+}$with $\mathcal{I} \neq A$, there exists $\mathcal{J}$ in $C$ such that $\mathcal{I} \circ \mathcal{J}$ is in $D(A)^{+}$and

$$
N(\mathcal{I} \circ \mathcal{J})<N(\mathcal{I})
$$

Then $A$ is a Krull domain and $C L(A)$ is a finite cyclic group. 
Proof. As we saw in Theorem 3, the existence of a norm map on $D(A)$ guarantees the ascending chain condition on the elements of $D(A)^{+}$. To show that $D(A)$ is a group it suffices to show that $C L(A)=\left\{C^{(-k)}\right\}_{k=1}^{\infty}$, because in particular the principal class is of the form $C^{(-k)}$ for some positive integer $k$, i.e. $C L(A)$ is a finite cyclic group from which it follows that $D(A)$ is a group.

If $\mathcal{I} \neq A$ is in $D(A)^{+}$we need to show that there is a positive integer $k$ so that $\mathcal{I}$ is in $C^{(-k)}$ and we will proceed by induction on $N(\mathcal{I})$. If any $\mathcal{J} \neq A$ in $D(A)^{+}$ is in $C^{(-k)}$ for some positive integer $k$ whenever $N(\mathcal{J})<N(\mathcal{I})$, then choose $\mathcal{B}$ in $C$ so that $\mathcal{I} \circ \mathcal{B}$ is in $D(A)^{+}$and $N(\mathcal{I} \circ \mathcal{B})<N(\mathcal{I})$. Now if $\mathcal{I} \circ \mathcal{B}=A$, then $\mathcal{I}$ is in $C^{(-1)}$ but if $\mathcal{I} \circ \mathcal{B} \neq A$, then by the induction hypothesis there exists a positive integer $k$ so that $\mathcal{I} \circ \mathcal{B}$ is in $C^{(-k)}$ and thus $\mathcal{I}$ is in $C^{(-k-1)}$.

Theorem 5. If $A$ is a Krull domain with a prime divisorial ideal in every divisorial ideal class, then $C L(A)$ is a finite cyclic group if and only if there is a norm $N$ on $D(A)$ and a class $C$ in $C L(A)$ with the following property: given $\mathcal{I}$ in $D(A)^{+}$with $\mathcal{I} \neq A$, there exists $\mathcal{J}$ in $C$ such that $\mathcal{I} \circ \mathcal{J}$ is in $D(A)^{+}$and

$$
N(\mathcal{I} \circ \mathcal{J})<N(\mathcal{I}) .
$$

Proof. Theorem 3 gives the proof of this equivalence in one direction. Suppose that $A$ is a Krull domain with a prime divisorial ideal in every divisorial ideal class and that $C L(A)$ is a finite cyclic group. If $n$ is the order and $C$ a generator of $C L(A)$, then $C L(A)=\left\{C^{(0)}, C, C^{(2)}, C^{(3)}, \ldots, C^{(n-1)}\right\}$. If $\mathcal{P}$ is a prime divisorial ideal and $\mathcal{P}$ is in $C^{(k)}, 0 \leq k \leq n-1$, then we set $N(\mathcal{P})=2^{n-k}$. Now setting $N(A)=1$ and extending $N$ to all of $D(A)$ by multiplicativity, it is clear that $N$ is a norm on $D(A)$. Suppose that $\mathcal{I}$ is in $D(A)^{+}$and $\mathcal{I} \neq A$; then we can write $\mathcal{I}=\mathcal{I}_{0} \circ \mathcal{P}$, where $\mathcal{I}_{0}$ is in $D(A)^{+}$and $\mathcal{P}$ is a prime divisorial ideal. If $\mathcal{P}$ is in $C^{(k)}, 0 \leq k \leq n-2$, we choose a prime divisorial ideal $\mathcal{Q}$ in $C^{(k+1)}$; then we have that $\mathcal{P}^{(-1)} \circ \mathcal{Q}$ is in $C$, $\mathcal{I} \circ \mathcal{P}^{(-1)} \circ \mathcal{Q}$ is in $D(A)^{+}$and $N\left(\mathcal{I} \circ \mathcal{P}^{(-1)} \circ \mathcal{Q}\right)=N\left(\mathcal{I}_{0} \circ \mathcal{Q}\right)<N(\mathcal{I})$. Finally if $\mathcal{P}$ is in $C^{(n-1)}$, then $\mathcal{P}^{(-1)}$ is in $C, \mathcal{I} \circ \mathcal{P}^{(-1)}=\mathcal{I}_{0}$ is in $D(A)^{+}$and $N\left(\mathcal{I}_{0}\right)<N(\mathcal{I})$.

Recall that a Dedekind domain is an integral domain $A$ with the property that $\mathcal{I}(A: \mathcal{I})=A$ for every nonzero ideal $\mathcal{I}$ of $A$. In this case $D(A)=M(A)$ is a group and the binary operation $\circ$ is just ideal multiplication.

Corollary 1. If $A$ is the ring of integers in an algebraic number field, then $C L(A)$ is a finite cyclic group if and only if there is a norm $N$ on $M(A)$ and a class $C$ in $C L(A)$ with the following property: given $\mathcal{I}$ in $M(A)^{+}$with $\mathcal{I} \neq A$, there exists $\mathcal{J}$ in $C$ such that $\mathcal{I} \mathcal{J}$ is in $M(A)^{+}$and

$$
N(\mathcal{I} \mathcal{J})<N(\mathcal{I}) .
$$

Proof. This result follows from Theorem 5, since a generalization of Dirichlet's theorem on primes in an arithmetic progression is that (see [5]) there is a prime ideal in every ideal class.

If $A$ is a Krull domain, then it is known (see [2]) that the polynomial domain in one variable $X$ over $A$, denoted by $A[X]$, is not only a Krull domain but every element of $C L(A[X])$ contains a prime divisorial ideal of $A[X]$.

Corollary 2. If $A$ is an integral domain, then $A$ is a Krull domain with $C L(A)$ finite cyclic if and only if there is a norm map $N$ on $D(A[X])$ and a class $C$ in 
$C L(A[X])$ with the property: given $\mathcal{I}$ in $D(A[X])^{+}$with $\mathcal{I} \neq A[X]$, there exists $\mathcal{J}$ in $C$ such that $\mathcal{I} \circ \mathcal{J}$ is in $D(A[X])^{+}$and

$$
N(\mathcal{I} \circ \mathcal{J})<N(\mathcal{I})
$$

Proof. It is proven in Fossum (see [2]) that $A[X]$ is a Krull domain if and only if $A$ is a Krull domain. Further if $A$ is a Krull domain, then $C L(A)$ is isomorphic to $C L(A[X])$. Now, by Theorem 14.3 on page 63 of Fossum (see [2]), if $A$ is a Krull domain, then there is a prime divisorial ideal of $A[X]$ in every element of $C L(A[X])$. Thus our result follows from Theorems 4 and 5 above.

If $A$ is a factorial domain or an almost factorial domain, then it is clear from the proofs of Theorems 2 and 3 that any norm on $D(A)$ will satisfy the condition of those theorems. However if $A$ is a Krull domain with a prime divisorial ideal in every divisorial ideal class, then it is not true that any norm on $D(A)$ will satisfy the condition of Theorem 5 .

Example 1. Let $A=Z[\sqrt{34}]$. Of course $A$ is a Dedekind domain and $C L(A)$ is cyclic of order 2 (see [1]). Further because $A$ is the ring of integers in an algebraic number field there is a prime ideal in every ideal class. We claim that the usual norm map on $D(A)=M(A)$ does not satisfy the condition of Theorem 5 . To that end we note that the $Z$-module $[3,4+\sqrt{34}]$ is a nonprincipal ideal and so it determines a class $C$ that generates $C L(A)$ as a cyclic group. The ideal $\mathcal{I}=(6+\sqrt{34})=$ $A(6+\sqrt{34})$ is principal but it is not equal to $A$. If there were an ideal $\mathcal{B}$ in $C$ so that $\mathcal{I B}$ is in $M(A)^{+}$and $N(\mathcal{I B})<N(\mathcal{I})$, then $\mathcal{B}=[3,4+\sqrt{34}] x$, where $x$ is in $K^{*}$, $(6+\sqrt{34})[3,4+\sqrt{34}] x \subseteq A$ and $|N(x)|<1 / 3$. Because $(6+\sqrt{34})[3,4+\sqrt{34}]=[6,4+$ $\sqrt{34}]$, we have that $x$ is a nonzero element of $(A:[6,4+\sqrt{34}])=[1,(4-\sqrt{34}) / 6]$, in which case $6 x$ is in $[6,4-\sqrt{34}]$ and $|N(6 x)|<12$. However we must then have that $|N(6 x)|=6$ and so it follows that $[6,4-\sqrt{34}]=(6 x)$, i.e. $[6,4-\sqrt{34}]$ is a principal ideal, which we know is not true.

\section{REFERENCES}

1. Harvey Cohn, Advanced number theory, Dover, 1980. MR 82b:12001

2. R. M. Fossum, The divisor class group of a Krull domain, Ergeb. Math. Grenzgeb. (3), bd. 74, Springer, Berlin, 1973. MR 52:3139

3. A. Fröhlich and M. J. Taylor, Algebraic number theory, Cambridge Univ. Press, Cambridge, No. 27, 1993. CMP 9311

4. Helmut Hasse, Über eindeutige Zerlegung in Primelemente order in Primehuapideale in Integritatsbereichen J. Reine Angew. Math. 159 (1928).

5. Gerald J. Janusz, Algebraic number fields, Academic Press, New York, 1973. MR 51:3110

6. Nathan Jacobson, Basic algebra I, second ed., Freeman, New York, 1985. MR 86d:00001

7. C. S. Queen, Euclidean like characterizations of Dedekind, Krull and factorial domains, J. Number Theory 47 (1994). MR 95f: 13025

8. G. Rabinowitsch, Eindeutigkeit der Zerlegung in Primzahlfaktoren in quadrat ischen Zahlkörpern, J. Reine Angew. Math 142 (1913), 153-164.

9. P. Samuel, Lectures on unique factorization domains, Tata Institute of Fundamental Research, Bombay, 1964. MR 35:5428

Department of Mathematics, Christmas-Saucon Hall, Lehigh University, 14 E. Packer Avenue, Bethlehem, Pennsylvania 18015

E-mail address: csq0@lehigh.edu 